\title{
QKD: Recovering Unused Quantum Bits
}

\author{
Abdulla M. Abu-Ayyash*, Naim Ajlouni** \\ *Arab Academy for BFS, **Al Balqa Electonic Academy \\ *abuayash@cbj.gov.jo,**najlouni@bea.edu.jo
}

\begin{abstract}
Practical quantum key distribution gain is usually less than the theoretical limit, this is due to the imperfection of physical devices, quantum channels, and the lost qubits due to test carried out for for the identification of the presence of an eavesdropper and the consequence reduction of obtained knowledge. In this paper a technique is presented to recover some of the unused qubits which result in an increase in the gain of the QKD protocols, in this research it was found that for the BB84 the recovered qubits can be as much as $11 \%$ of the initial key bits. This technique is based on discovering the relationship between the initial key bits and the original random bits which will aid in the recovery process of the unused qubits ( unused qubits are the bits which are assumed to be lost at the start of the process due to the random process and for $\mathrm{BB84}$ it is assumed to be around $50 \%$ of the generated key bits).
\end{abstract}

\section{INTRODUCTION}

Quantum key distribution (QKD) protocols $[1][2][3][4][5][6][7][8][9]$ intention is to agree on a key over quantum and non-quantum channels in order to use the proved [11][12] secured cryptographic encryption protocol named Vernam's one-time pad, Unfortunately the key size generated is smaller than the generated pulses of quantum sates (photons) in a multiple of magnitudes. In a study using BB84 protocol [10] the ratio between the generated pulses and the finally agreed key is around 950:1, and the ratio between the received qubits to the final key is about $19 \%$, it is even less than that for B92 [2] and E91 [3]. To increase the size of the key, one may use the pseudo-random bit generation (PRBG) algorithms [13][14] with the seed being the key agreed on by the two parties. It should be noted that not any PRBG is suitable for cryptographic use, and only if PRBG passes the next-bit prediction test then, it is called cryptographically secured pseudo-random bit generator (CSPRBG) [13]. In section III we will discus the reasons for the loss of QKD protocols; section IV discuses the proposed method of recovering unused quantum bits (RUQB), section $\mathrm{V}$ concludes the paper.

\section{QuAntum Key Distribution (QKD)}

The basic element of quantum key distribution will be illustrated using the the original four - state QKD protocol developed by Bennett and Brassard in 1984 known as "BB84" protocol [10]. In this case it is assumed that the individual photons serve as the quantum bits for the protocol, or more precisely, the polarization states of individual photons. The protocol start by one of the parties transmitting a sequence of photons to the other party. The parties publicly agree to make use of the two distinct polarization bases which are chosen to be maximally non-orthogonal.In a completely random order, a sequence of photons are prepared in states of definite polarization in one or other of the two chosen bases and transmitted by one of the parties to the other through a channel that preserves the polarization. The photons are measured by the receiver in one or the other of the agreed upon bases, again chosen in a completely random order. The choices of bases made by the transmitter and receiver thus comprise two independent random sequences. Since they are independent random sequences of binary numbers, about half of the basis choices will be the same and are called the "compatible" bases, and the other half will be different and are called the "incompatible" bases. The two parties compare publicly, making use for this purpose of a classical communication channel, the two independent random sets of polarization bases that were used, without revealing the polarization states that they observed.

\section{REASONS FOR LOSS}

Gilbert et al [15] has shown that the state function for a coherent state produced by the laser pulse is given by

$$
|\psi\rangle=\sum_{l=0}^{\infty} \sqrt{e^{-\mu} \frac{\mu^{l}}{l !}} e^{i l \psi}|l\rangle
$$

where $e^{i l \psi}$ is the phase factor, $\psi$ is the quantum mechanical phase. In this case both can be neglected, as they have no effect on the probability of the number of photons $l$ generated, $\mu$ is defined as the expectation value of the number operator, and in practice the mean number of photons per pulse. Therefore it is clear that the number of photons produced in each pulse is thus characterized by a Poisson distribution.

From equation (1) the probability a state $|l\rangle$ is being generated has the value of $e^{-\mu} \frac{\mu^{l}}{l !}$, if we denote it by $\chi(\mu, l)$, which is the probability that a laser pulse (in a stream of pulses characterized by $\mu$ ) will contain exactly $l$ photons and thus $\chi(\mu, l)=e^{-\mu} \frac{\mu^{l}}{l !}$, and the probability of generating more than two photons is $\psi_{\geq 2}(\mu)=(1-\chi(\mu, 0)-\chi(\mu, 1))=1-e^{-\mu}-\mu e^{-\mu}$ . Therefore in order to prevent an eavesdropper from beam splitting the transmitted pulse, it is necessary to reduce the probability of producing more than one photon, in order to do so we have to select $\mu$ to be very small, say around $\mu=0.1$, hence the probability of a pulse containing two or more photons will be $\psi_{\geq 2}(0.1)=1-e^{-0.1}-0.1 e^{-0.1}=0.0047$, and the probability of producing only one photon is $\chi(0.1,1)=0.09 \approx 0.1$. 
The efficiency of QKD gain is affected by the amount of attenuation $\alpha$ that characterizes the propagation loss of the quantum channel, the efficiency of the detector of Bob $\eta$, the probability of the detector indicating a false click when no event has occurred (dark count probability) $r_{d}$, as well as the protocol itself.

Error correction process which is carried through the non-quantum channel can reveal more information \{about\} an eavesdropper that needs to be eliminated or reduced, Another process named privacy amplification which is aimed to reduce the information that an eavesdropper may gain without the legitimate parties noticing that (by beam-splitting or intercept/resend methods), which results in yet more bits being eliminated, hence more loss.

For example BB84 probability of matching the bases is $50 \%$ from all received pulses, test for Eve takes around 50\% from the matched bases which leaves only $25 \%$ to start error correction and privacy amplification. This will only leave around $19 \%$. B92 probability of agreeing on the bit is $25 \%$, testing for Eve also takes $50 \%$ which leaves only $12.5 \%$ to start error correction and privacy amplification, E91 has the same problem.

A number of researchers have already tried to solve this problem in a multi-dimensional space; one dimension is enhancing the physical devices, channels, parameters and implementation, such as single photon generators and detectors [20], [19], [22], another dimension is changing or enhancing the protocols [4], [5], [9], a third is to try and increase the information content in the quantum states used in Qutrit and four-state quantum elements [18], [21]. A forth is using other quantum phenomena similar to EPR and entangling atoms with photons [17], [3], [21].

The purpose of this research is to develop a method for recovering unused quantum bits (RUQB). This will enhance efficiency of QKD, since the enhancement can be used for any QKD, the examples, calculations, and implementations in this research will be based on BB84 since it is the first, simplest and well known QKD algorithm.

\section{Recovering UnUSED QuANTUm BITS (RUQB)}

Most QKD algorithms starts by using random (not a pseudorandom) numbers generated by any physical phenomena, allot of such random numbers are used for testing the presence of an eavesdropper which reduces the gain of the protocol. RUQB is based on the idea of recovering unused bits, the actual recovery process will concentrate on recovering such random numbers by making use of the agreed bits from the random number list. The following will emphasize RUQB:

1) Alice generate a set of $n$ random bits $A=$ $\left\{a_{0}, a_{1}, \cdots, a_{n-1}\right\}, n=2^{l-1}$, where $l \in Z$.

2) Alice encode the bits into quantum states to have $Q=$ $\left\{\left|q_{0}\right\rangle,\left|q_{1}\right\rangle, \cdots,\left|q_{n-1}\right\rangle\right\}$ and sends the quantum states to Bob.
3) Bob measures each quantum state received based on any QKD method to obtain $B=\left\{b_{0}, b_{1}, \cdots, b_{n-1}\right\}$.

4) Alice and Bob start a sifting process based on the same QKD method result in $F$ (set of agreed on locations of bits).

5) Alice and Bob directly compare $\frac{|F|}{2}$ bits to estimate the number of errors $e_{T}$ and its percentage given by $\epsilon=$ $\frac{2 e_{T}}{|F|}$, if the percentage is more than a threshold $t$ they abort the protocol and start again, if not they start an error correction process to eliminate the set $E$ of bits to obtain the initial key string $K_{I}^{a}=\left\{a_{i} \mid \forall a_{i} \in A, i \in\right.$ $F-E\}, K_{I}^{b}=\left\{b_{i} \mid \forall b_{i} \in B, i \in F-E\right\}$, note that $\left|K_{I}^{a}\right|=\left|K_{I}^{b}\right|<n$.

6) RUQB: Alice construct a list $L$ and send it to Bob as follows:-

a) calculate the number of bits needed to represent any location of the originally (Alice) generated random bits $l=\left\lceil\log _{2} n\right\rceil+1$.

b) Alice chooses randomly a number 0 or 1 and denote it $R^{a}$.

c) if $R^{a}$ is 1 then reverse every bit in the $K_{I}^{a}$ (i.e. $0 \rightarrow 1,1 \rightarrow 0$, lets donate this by $\neg K_{I}^{a}$ ).

d) construct a set of bits from the initial key bits $S_{i}^{a}=$ $\left\{c_{j} \mid \forall c_{j} \in K_{I}^{a}, i \leq j<i+l\right\}, 0 \leq i \leq\left|K_{I}^{a}\right|-l$.

e) construct a temporary set of results $K_{T}^{a}=$ $\left\{\left(r_{i}, l_{i}, i\right)\right\}, 0 \leq i \leq\left|K_{I}^{a}\right|-l$ where $r_{i}$ is the result of XORing the elements of the set $S_{i}^{a}$ as shown in equation $2, l_{i}$ is the binary location calculated from set $S_{i}^{a}$ as

$$
\begin{aligned}
r_{i} & =\bigoplus_{\substack{c_{j} \in S_{i}^{a} \\
0 \leq j<l}} c_{j}, \\
l_{i} & =\left(\sum_{\substack{c_{j} \in S_{i}^{a} \\
0 \leq j<l}} c_{j} 2^{j}\right) \quad \bmod n,
\end{aligned}
$$

f) construct an indexing list $L=\left\{i \mid \forall\left(r_{i}, l_{i}, i\right) \in\right.$ $\left.K_{T}^{a}, a_{l_{i}}=r_{i}, l_{i} \notin F \cup E\right\}$.

$$
a_{l_{i}} \in\left\{\begin{array}{cc}
A & \text { if } R^{a}=0 . \\
\neg A & \text { if } R^{a}=1
\end{array}\right.
$$

g) Alice calculate $r=\bigoplus_{i \in L} a_{i}, a_{i} \in A$.

h) if $r \neq R^{a}$ then modify $L$ by selecting and deleting an item $i_{l} \in L$ that after recalculating $r$ result in $r=R^{a}$.

i) Alice key is $K^{a}=\left\{k_{i}\right\}$, such that

$$
k_{i}= \begin{cases}a_{i} & \forall a_{i} \in\left\{\begin{array}{cc}
A & \text { if } R^{a}=0 . \\
\neg A & \text { if } R^{a}=1
\end{array}, i \in F-E .\right. \\
r_{i} & \forall\left(r_{i}, l_{i}, i\right) \in K_{T}^{a}, \forall i \in L, l_{i} \notin F\end{cases}
$$

7) once Bob receives $L$ he will calculate the following:-

a) $l=\left\lceil\log _{2} n\right\rceil+1$.

b) Bob calculate $R^{b}=\bigoplus_{i \in L} b_{i}, b_{i} \in B$.

c) if $R^{b}$ is 1 then reverse every bit in the $K_{I}^{b}$.

d) $S_{i}^{b}=\left\{c_{j} \mid \forall c_{j} \in K_{I}^{b}, i \leq j<i+l\right\}, \forall i \in L$. 
e) $K_{T}^{b}=\left\{\left(r_{i}, l_{i}, i\right)\right\}, \forall i \in L$ where

$$
\begin{aligned}
r_{i} & =\bigoplus_{\substack{c_{j} \in S_{i}^{b} \\
0 \leq j<l \\
0, j}} c_{j} \\
l_{i} & =\left(\sum_{\substack{c_{j} \in S_{i}^{b} \\
0 \leq j<l}} c_{j} 2^{j}\right) \bmod n
\end{aligned}
$$

f) $K^{b}=\left\{k_{i}\right\}$, such that

$$
k_{i}=\left\{\begin{array}{cc}
b_{i} \quad \forall b_{i} \in\left\{\begin{array}{cc}
B & \text { if } R^{b}=0 \\
\neg B & \text { if } R^{b}=1
\end{array}, i \in F-E .\right. \\
r_{i} \quad \forall\left(r_{i}, l_{i}, i\right) \in K_{T}^{b}, \forall i \in L, \\
l_{i} \notin F .
\end{array}\right.
$$

Note that the gain $G$ can be calculated as

$$
G=\left(\frac{|F|}{2}-|E|-l\right) P_{c l u} P_{c v}\left(1-P_{l_{r}}\right)
$$

where $|F|$ the size of the sifted key ffor BB84 it is approximately $\frac{n}{2}$ and B92 is approximately $\left.\frac{n}{4}\right\},|E|$ is the expected number of bits to be eliminated if there is an error, in the best case the algorithm eliminates only the errors, but this is not the case ${ }^{1}, l=\left\lceil\log _{2} n\right\rceil+1, P_{c l u}$ the probability that the calculated location of RUQB will not be used within the initial key \{for BB84 approximately it is $\frac{1}{2}$, for B92 $\frac{3}{4}$ \}, $P_{c v}$ probability of correct value found at unused location and it is approximately $\frac{1}{2}, P_{l_{r}}$ is the probability of redundant locations which is random generator dependent.

For large value of $n$ (i.e. $n \gg l$ ), $G$ is approximated as

$$
G \simeq\left(\frac{|F|}{2}-|E|\right) P_{c l u} P_{c v}\left(1-P_{l_{r}}\right)
$$

In this paper RUQB will be discussed and present using two different working conditions; with or without errors.

\section{A. RUQB with the absence of errors}

if we assume that the quantum channel is error free and Eve is not eavesdropping on the quantum channel then $|E|=0$, therefore $G$ is given by equation 10 .

$$
G \simeq\left(\frac{|F|}{2}\right) P_{c l u} P_{c v}\left(1-P_{l_{r}}\right)
$$

Now BB84 gain is estimated as given by

$$
G_{B B 84} \simeq\left(\frac{1-P_{l_{r}}}{16}\right) n
$$

Maximum gain can be obtained when their is no redundancy 2 . BB84 maximum gain is about $6.25 \%$ of $n$.

\footnotetext{
${ }^{1}$ it depends on the algorithm used to eliminate those errors, Shannon estimated the minimum limit for the number of bits that need to be communicated to eliminate errors based on the probability of error for each transmitted bit $\epsilon$ to be $n h(\epsilon)$, some algorithms need $x n h(\epsilon)$, where $x \geq 1[15]$.

${ }^{2}$ random generators generate $n$ different patterns of size $l$.
}

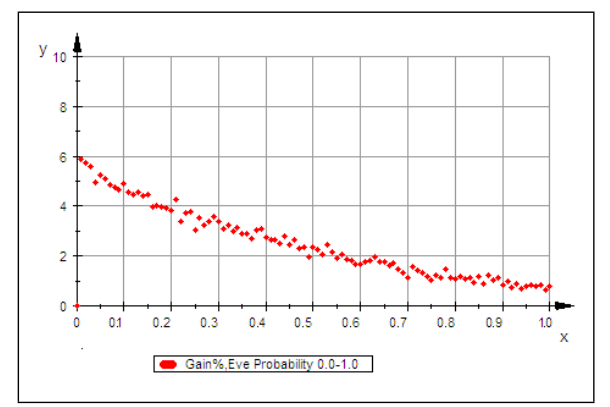

Fig. 1. The effect of eavesdropping (with probability range between $0.0-1.0$ ) on the gain G, sample 1024 qubits .

Table I lists an average of 100 run for each selected $n$, figure 2 and 3 shows the gain $G$ and the probability of redundancy $P_{l_{r}}$ of runs of 512 qubit. Figure 4 and 5 is for 16384 qubits. Based on multiple runs and the constant value of $G \%=\frac{G}{n} 100 \% \approx 5.5 \%$ (the last column in Table I), $P_{l_{r}}$ was estimated to be around $12 \%$ (not including the numbers that are not $2^{l-1}$ ).

Note that the probability of redundant locations $P_{l_{r}}$ varies from $2 \%$ to $27 \%$ for a list of 512 qubits while groups around 11.5\% for 16384 qubits (see Figures 3 and 5). Figure 5 shows a narrow range for the probability $P_{l_{r}}$ (between $10 \%$ to $14 \%$ ).

\section{B. RUQB With the presence of errors}

If the quantum channel is not error-free and/or Eve is eavesdropping on the quantum channel then errors will be within the sifted key and they should be eliminated, Shannon theorem estimates the minimum number of bits to be eliminated due to errors/Eve by $|E|=\frac{|F|}{2} h\left(\frac{2 e_{T}}{|F|}\right)$, where $h(\epsilon)=-\left(\epsilon \log _{2} \epsilon+(1-\epsilon) \log _{2}(1-\epsilon)\right)$, therefore $G$ is estimated by

$$
G \simeq\left(\frac{|F|}{2}\left(1-h\left(\frac{2 e_{T}}{|F|}\right)\right)\right) P_{c l u} P_{c v}\left(1-P_{l_{r}}\right)
$$

Now under the same conditions BB84 gain is estimated by

$$
G_{B B 84} \simeq\left(\frac{\left(1-P_{l_{r}}\right)(1-h(\epsilon))}{16}\right) n
$$

Figure 1 shows the effect of eavesdropping with different probabilities between 0.0 (no eavesdropping) up to 1.0 (full eavesdropping) on the RUQB gain, which induce errors as if the quantum channel has errors $\epsilon$ between 0.0 up to 0.25 , note the loss of the gain with the increase of errors.

\section{RUQB Variable Selection}

1) The selection of $l=\left\lceil\log _{2} n\right\rceil+1$ is due to the fact that the same location $l_{i}$ calculated using equation (7) can have two possible values for $r_{i}$ (0 or 1) with equal probability (not to be binary representation dependant).

2) the selection of $n=2^{l-1}$ is due to the fact that location $l_{i}$ has the same probability of being found within the original list of size $n$ after taking the modulus $n$ and 


\begin{tabular}{|c|c|c|c|c|c|c|c|c|}
\hline No. & $n$ & $l$ & $\overline{|F|}$ & $\overline{\overline{P_{c l u}}}$ & $\overline{P_{c v}}$ & $\bar{G}$ & $\overline{P_{l_{r}}}$ & $\bar{G} \%$ \\
\hline 1 & 512 & 10 & 246 & 0.495 & 0.500 & 27 & 0.101 & 5.27 \\
\hline 2 & 1024 & 11 & 502 & 0.511 & 0.500 & 55 & 0.130 & 5.37 \\
\hline 3 & 2048 & 12 & 1017 & 0.513 & 0.497 & 112 & 0.136 & 5.46 \\
\hline 4 & 4096 & 13 & 2035 & 0.511 & 0.499 & 224 & 0.136 & 5.46 \\
\hline 5 & 8192 & 14 & 4078 & 0.499 & 0.501 & 452 & 0.115 & 5.51 \\
\hline 6 & $10240 *$ & 15 & 5102 & 0.498 & 0.502 & 542 & 0.150 & 5.29 \\
\hline 7 & 16384 & 15 & 8178 & 0.499 & 0.501 & 906 & 0.114 & 5.53 \\
\hline 8 & $102400 *$ & 18 & 51067 & 0.503 & 0.493 & 5430 & 0.140 & 5.30 \\
\hline 9 & 131072 & 18 & 65646 & 0.498 & 0.503 & 7299 & 0.112 & 5.56 \\
\hline average & & & & 0.503 & 0.500 & & 0.120 & 5.48 \\
\hline
\end{tabular}

AVERAGE OF 100 RUNS FOR EACH $n$, *NUMBERS ARE NOT $2^{l-1}$ AND NOT INCLUDED IN AVERAGE.

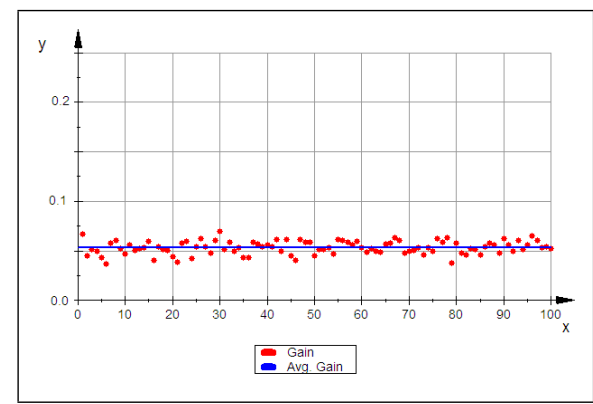

Fig. 2. 100 run of $2^{9}=512$ qubits, average $\bar{G}=5.27 \%$.

so minimizing the probability of redundant location calculation and this will increase the gain $G$ for the same $l$ (compare item 6 and 8 with 7 and 9, respectively in table I).

3) the introduction of $R^{a}$ is to decrease the information that Eve may gain by eavesdropping, as it will not be able to identify the key bits as and when they are in their reverse order.

\section{Security of $R U Q B$}

In general cases Eve uses a beam-splitting method to obtain information about QKD based on the probability of more than one photon contained within the photon pulse, which is related to the mean number of photons per pulse $\mu$ that characterise the photon pulse generator. Intercept/resend method will allow Eve to have access to what Alice or Bob sent or received respectively, as a result Eve will at the some times introduce errors due to her interception.

Based on the value of both $\mu$ and errors $\epsilon$, one can estimate

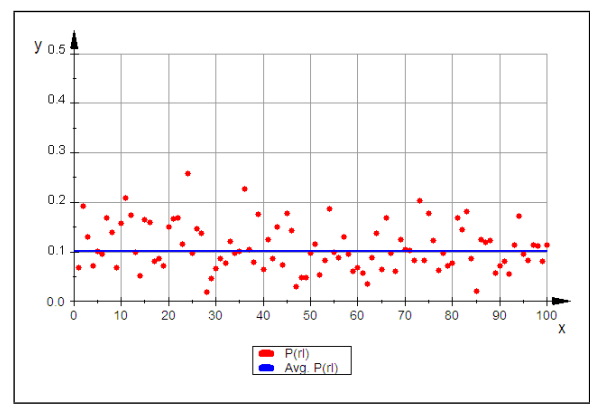

Fig. 3. 100 run of $2^{9}=512$ qubits, average $\overline{P(r l)}=10 \%$.

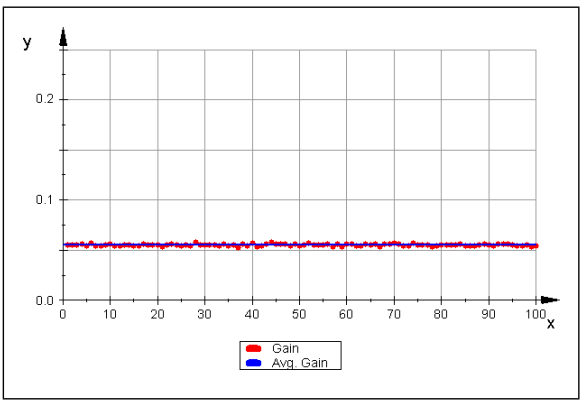

Fig. 4. 100 run of $2^{14}=16384$ qubits, average $\bar{G}=5.53 \%$.

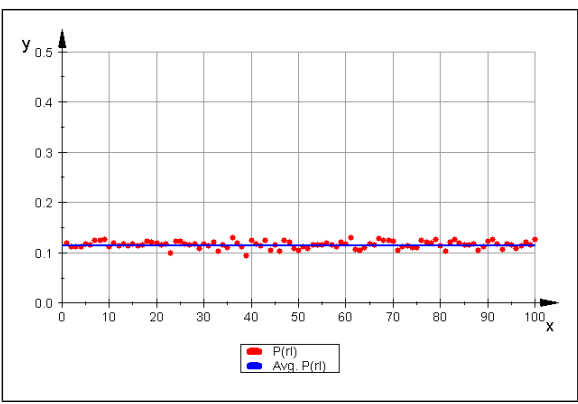

Fig. 5. 100 run of $2^{14}=16384$ qubits, average $\overline{P(r l)}=11.4 \%$.

the information leaked that need to be eliminated by privacy amplification. In [10] this value was estimated to be:

$$
I_{e}=|F|(\mu+2 \sqrt{2} \epsilon)+5 \sqrt{|F|(\mu(1-\mu)+(4+2 \sqrt{2}) \epsilon)}
$$

In this case it is necessary to identify whether RUQB has caused more information to be leaked about the initial and final keys or not. To answer this, we need to assume that RUQB will allow Eve to be in possession of a set $L$ which contains the index of the locations within the initial key, which will contribute to more bits within the final key. This set is of no value to Eve unless any or both of the following conditions hold:-

1) All the locations within the initial key that happen to be within $L$ is known. (i.e. for BB84 a specific $11 \%$ of the the initial key is known by Eve).

2) A known sequence of $l$ or more bits within the initial key and the start of this sequence must be within $L$ (i.e. with a probability of 0.11 this sequence contribute to new bit).

\section{CONCLUSION}

Most QKD protocols uses random generated bits or qubits and suffer from a gain problem that need to be solved; in this paper RUQB introduced a new method that is able to recover some of the unused quantum bits.

The recovery is achieved by re-examining the initial $50 \%$ of the bits assumed to be lost. This is based on the process of finding the relationship between the random bits generated.

The effect of this recovery as was seen causes a big improvement if it is compared to the percentage of bits which 
are assumed to be received correctly, which is in the case of the BB84 estimated to be around $19 \%$ and in the best case around $25 \%$. Therefore the recovered qubits (5.5\%) will improve the above results up to $24.5 \%$ in the worst case to $30.5 \%$ in the best case. Therefore the recovery process improves the gain by a minimum of $35 \%$.

Also the complexity of RUQB is given by $O\left(\frac{|F| \log n}{2}\right)$, while it is about $O\left(\frac{n \log n}{4}\right)$ for the BB84.

\section{REFERENCES}

[1] C.H. Bennett and G. Brassard, Quantum cryptography: Public key distribution and coin tossing, Proceedings of the IEEE International Conference on Computers, System, and Signal Processing, Bangalore, India (IEEE, New York. 1984), pp. 175-179.

[2] Charles Bennett, Quantum Cryptography using any two non orthogonal states.IBM Research Division T J Watson Research Center Yorktown Heights, phy. Rev Lett vol 68 num 21 p3121-3124.

[3] A. K. Ekert, Phys. Rev. Lett. 67, 661 (1991).

[4] Subhash Kak, A Three-Stage Quantum Cryptography Protocol, arXiv:quant-ph/0503027v2 2 Mar 2005, Foutions of Physics Letters 19 (2006), 293-296.

[5] Yoshito Kanamori, Seong-Moo Yoo and Mohammad Al-Shurman, A Quantum No-Key Protocol for Secure Data Communication, 43rd ACM SE Conference, March 18-20, 2005, Kennesaw.

[6] Kim Bostrom, TimoFelbinger, Deterministic Secure Direct Communication Using Entanglement, arXiv:quant-ph/0209040 v2 13 Oct 2002.

[7] Marco Lucamarini and Stefano Mancini, Secure Deterministic Communication Without Entanglement, arXiv:quant-ph/0405083 v3 24 May 2004.

[8] Won Young H0-wang, In Gyu Koh and Yeong Deok Han, Quantum cryptography without public announcement bases, quant-ph/9702009 v5 12 May 97.

[9] Jonathan Barrett,Lucien Hardy,and Adrian Kent, No Signalling and Quantum Key Distribution, arXiv:quant-ph/0405101 v329 Apr 2005

[10] C. H. Bennett, G. Brassard, L. Salvail, and J. Smolin, Experimental quantum cryptography J. Cryptology 5, 3 (1992).

[11] CE Shannon, A Mathematical Theory of Communication, in Bell Systems Technical Journal, v 27 (1948), pp 379n423, 623n656.

[12] CE Shannon, Communication Theory of Secrecy Systems, in Bell Systems Technical Journal, v 28 (1949), pp 656n715.

[13] Alfred Menezes, Paul van Oorschot, and Scott Vanstone, Handbook of Applied Cryptography, CRC Press, 1997.pp170-171.

[14] Oded Goldreich, Foundations of Cryptography Fragments of a Book, February 1995, http://theory.lcs.mit.edu/ oded/frag.html.

[15] [Gilbert00]G. Gilbert, M. Hamrick, Practical Quantum Cryptography: A Comprehensive Analysis, Mitre Technical Report arXiv:quant$\mathrm{ph} / 0009027$ v5 4 Sep 2000.

[16] R.F. Blute and Prakash Panangadeny, Fock Space: A Model of Linear Exponential Types,

[17] A. Einstein, B. Podolsky and N. Rosen, Can Quantum-Mechanical Description of physical reality be considered complete?, Institute for advanced study, Princeton, new jersey, Vol 47 p777-780 (1935) .

[18] Simon Groblacher Thomas Jennewein, Alipasha Vaziri, Gregor Weihs and Anton Zeilinger, Experimental Quantum Cryptography with Qutrits,arXiv:quant-ph/0511163 v1 16 Nov 2005.

[19] C. Santori D. et al, Generation of single photons and correlated photon pairs using InAs quantum dots, Fortschr. Phys. 52, No. 11-12, 1180 - 1188 (2004) / DOI 10.1002/prop.200410188, WILEY-VCH Verlag GmbH \& Co. KGaA,Weinheim.2004

[20] [Chou04] C.W. Chou, S.V. Polyakov, A. Kuzmich, H.J. Kimble, Singlephoton generation from stored excitation in an atomic ensemble. Phys. Rev. Lett. 92, 213601 (2004).

[21] Le-Man Kuang and Lan Zhou1, Generation of atom-photon entangled states in atomic Bose-Einstein condensate via electromagnetically induced transparency, arXiv:quant-ph/0402031 v1 4 Feb 2004

[22] S. Tisa, A. Tosi and F. Zappa, Fully-integrated CMOS single photon counter, Politecnico di Milano, Dipartimento di Elettronica e Informazione, Piazza Leonardo da Vinci 32, I-20133 Milano, Italy, Optical Society of America, 2007. 